\title{
The Upward Strengthening Hypothesis
}

Welcome back to Ten Lectures on Diachronic Construction Grammar. This is the ninth lecture. In the last lecture, I discussed unidirectionality in language change and the idea that grammatical semantic change proceeds in one direction only, from more concrete, more specific meanings to more abstract meanings. The empirical studies that I discussed were designed to test whether this particular characteristic of language change could be explained through the cognitive process of priming. Since many processes of language change in grammaticalization are clearly asymmetrical, it is a very tempting idea that these processes of change are also triggered by a psychological mechanism that is asymmetrical, as in the case of priming relations that go in one direction only. I explained that the predictions of the asymmetric priming hypothesis ultimately could not be substantiated, but there is still reason to believe in the basic idea that historical processes of language change are to be explained in terms of cognitive processes that are at work in the here and now. When I talk to you, certain cognitive processes are active, and those are the same processes that are responsible for language change in the long run. This basic idea is also of interest for my lecture today. The title for this lecture is "The upward strengthening hypothesis". I have used this term in a paper of mine that was published in 2015 in the journal Cognitive Linguistics. I used it in order to combine several notions that are widely shared in Construction Grammar and cognitive linguistics in general, but that have not been put together in quite the same way that I thought would be insightful. Let me explain what I mean.

At the heart of the upward strengthening hypothesis is the notion that concrete usage events send activation through the network of construction that is your knowledge of language. As you hear me talk as I do right now, the words that you hear activate your mental representations of constructions in your knowledge of language.

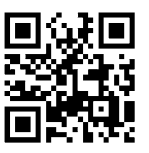

All original audio-recordings and other supplementary material, such as any hand-outs and powerpoint presentations for the lecture series, have been made available online and are referenced via unique Dor numbers on the website www.figshare.com. They may be accessed via this QR code and the following dynamic link: https://doi.org/10.6o84/mg.figshare.13691269. 
Let's take a simple example. When I say the word hypothesis, then the node in your network of constructions that corresponds to that word hypothesis is activated. When I say the upward strengthening hypothesis, there are four words that are activated, the, upward, strengthening and hypothesis, but at the same time, also a syntactic construction is activated, namely the "definite noun phrase" construction. The upward strengthening hypothesis instantiates a syntactic pattern and that pattern is a construction in your knowledge of language that is being activated at that point.

The upward strengthening hypothesis is thus based on the idea that all of your knowledge is a network of form-meaning pairs. It crucially relates to the concept of entrenchment. Mental representations of linguistic structures are strengthened if they are activated very often.

Over these past days, you've heard me pronounce certain words very often, as for instance Diachronic Construction Grammar. This particular phrase has been strengthened, it has been entrenched in your mind. The more often I pronounce a word or a construction, the more activation is sent to that particular node in your network of constructions, and the more entrenched that node becomes. The upward strengthening hypothesis relates to activation of your linguistic knowledge and the gradual entrenchment of that knowledge. That represents a broad consensus that we currently have in usage-based linguistics, but there is more to the upward strengthening hypothesis.

Another component of the hypothesis is what I presented as basic idea \#3, the idea that constructions vary in terms of their degrees of complexity and schematicity. When you hear me say the upward strengthening hypothesis, you hear the concrete words, the, upward, strengthening and hypothesis, which are constructions that are not schematic and not very complex. Those words are activated in your mind, and they become a little more entrenched. But you also registered that these words combine to form a definite noun phrase, which is a constructional schema, a construction that is both complex and schematic. What happens to that schema when you hear a new instance of it? The idea, reasoning from the basic principles of usage-based linguistics, would be also that schema is activated and strengthened as you hear me pronounce that phrase.

Your knowledge of language is organized in different layers of complexity and schematicity that characterize the nodes of the constructional network. Schematic constructions are higher up in the network and specific constructions are further down.

The question that is inherent the upward strengthening hypothesis ultimately is this one: When I hear a construction, a simple one like the word dog, does the activation that runs through my knowledge of language only go to 
that construction itself, or does it also strengthen more abstract constructions like the noun construction? Is there activation that starts with the $d o g$ and then flows up and strengthens a node that is higher up in the network?

Let's take another example. When I hear an expression such as the hypothesis, which is a bit more complex, as it consists of two words, does the activation go to that particular string only, or does it also go to the schematic construction that this string instantiates? That schema would be the definite noun phrase construction. We do not have to stop there. Does the hypothesis activate the definite noun phrase construction and in turn a general noun phrase construction? How far up does the activation go? That is the underlying question that I would like to address.

This question relates to one of the controversies that I mentioned on the first day, namely the controversy between the views of complete inheritance on the one hand and of redundant representations on the other. Just to refresh your memory, the relevant issue is whether speakers cognitively represent grammatical information just once, at the highest level of abstraction, or whether they represent it several times, across different layers of abstraction in the constructional network.

According to the view of complete inheritance, information is stored only once at the most abstract level, which is very economical and elegant, but which ultimately might not correspond to the psychological facts. The view of redundant representations, on the other hand, would hold that information is stored at several levels of abstraction, so that speakers remember forms that they technically they would not need to remember, such as the plural noun cats.

On the view of complete inheritance, the answer would be that cognitive strengthening affects all layers up to the most abstract construction, because hearing a phrase like the hypothesis would require you to look up certain pieces of information that are stored in the definite noun phrase construction. What does it mean to be definite? That is information that is stored in the definite noun phrase construction. When you hear the hypothesis, you would further need to access the general noun phrase construction in order to look up information such as that noun phrases can be subjects or objects in the clause. All of that information is only stored once, and as a hearer, you have to activate the respective level to find that information.

Let me say a few words about inheritance as it is normally understood in Construction Grammar. This concerns the relations between more abstract constructions and more concrete constructions.

Earlier, I have given you the example of an idiom such as kick the habit, which inherits information from transitive kick, which inherits information 
from the transitive construction, which further inherits information from the general verb phrase construction. On the complete inheritance view, lowerlevel constructions do not redundantly represent information that is inherited. Information that can be looked up does not have to be stored. By contrast, on the view of redundant representations, low-level constructions have rich representations.

Crucially, these two views of inheritance have different implications for the upward strengthening hypothesis. Namely, when you hear the hypothesis, according to the idea of complete inheritance, you have to access the definite noun phrase in order to look up features like definiteness, and you have to access the general schematic noun phrase construction in order to look up features that are common to all noun phrases. In other words, with regard to the upward strengthening hypothesis, the view of complete inheritance would imply that every time you hear a construction, activation spreads through the entire network all the way up to look up the most general features of that construction. Specifically, when you hear dog, also your representation of the noun construction becomes a bit more entrenched. When you hear the hypothesis, the general noun phrase construction becomes a little bit more entrenched. By contrast, the view of redundant representations would allow you to stop at a certain level, which already represents all the relevant features redundantly, so you hear dog, but nothing happens to the noun construction. Dog is a very frequent word that you have stored, so no further activation spreads to the noun construction as such.

Ineed to come back for a minute to the idea of constructionalization, which I want to contrast with the idea that I call upward strengthening. Just to remind us, constructionalization is the creation of a new form-meaning pair in the network of constructions. Constructionalization requires the repeated exposure to concrete tokens of language use. You hear a certain type of expression again and again, which then leads to the formation of a new abstract node in the network of constructions.

What I would like to stress is that constructionalization and upward strengthening are not the same phenomenon. Grammatical constructionalization is the establishment of a new node in the network, and upward strengthening is concerned with its subsequent entrenchment through the experience of language use. Crucially, the experience of a linguistic unit may strengthen not only a representation of that unit itself. When you hear a word or an expression, not only that expression itself, not only that construction itself may be strengthened, but also the more abstract schema that licenses that expression in the first place. The claim that I want to make in the following is that upward strengthening is a process of grammaticalization. 
Let me also stress that upward strengthening is not the same as entrenchment. Entrenchment, as it is typically understood, means that you hear a linguistic unit and the representation of that linguistic unit is strengthened in your mind. What I would like to discuss is how abstract patterns become entrenched.

Abstract patterns are not something that you hear. You hear strings of words, and the more you hear a certain string, the more routinized that string becomes, and the better you know it. But what about abstractions of these strings? Let's take an example. Take the expression kind of funny, which consists of three words that frequently occur together. Over time, the string may become entrenched in your mind, so that you recognize it as an idiomatic phrase of English. That string instantiates a more abstract schema, namely kind of $A D J$. Speakers can describe entities and events as kind of sad, kind of complicated, kind of expensive and so on and so forth. How does that abstract pattern become entrenched? Kind of can also appear with other types of phrases. The final element does not have to be an adjective. In the utterance "Well, he's kind of a jerk", the final element is a noun phrase, not an adjective.

Let us take a concrete example. You hear kind of funny and that speech event sends activation to that very expression. That exact string becomes more strongly represented in your mind. Let's for the moment assume that this speech event also passes on a certain measure of activation to a more abstract schema like kind of $A D J$. That would mean that this kind of phrase higher up in the network would become a little bit more entrenched. Going even one step further, if this the kind of ADJ schema is activated and passes on part of its activation to an even more abstract schema, that would lead to the entrenchment of an abstract constructional schema in your mind.

I have already mentioned the conflicting predictions of the complete inheritance view and the view of redundant representations. The view of complete inheritance invites an understanding of upward strengthening that I would like to call the "naive" strengthening hypothesis. What that would mean in practice is that every time you hear an expression, activation would spread upward, as far as it can possibly go. Let us take the expression You drive me crazy as an example. On the naive strengthening view, each component part of this would be strengthened, so You drive me crazy sends activation to each word that it contains: you, drive, me and crazy. As a construction, You drive me crazy would further be assumed to send upward activation to the drive crazy construction, which is more abstract. That construction does not only have you as a subject. It also allows for any kind of object, not just $m e$. On the naive strengthening view, we do not redundantly store information at lower levels of representation. The idiomatic drive crazy construction inherits information from the 
resultative construction, SUBJ VERB OBJ ADJP. That construction licenses not only "You drive your parents crazy", but also "You hammer the metal flat" and "He painted the wall blue". In order to process and understand You drive me crazy, we thus need to look up some information at the level of the $S U B J$ drive OBJ crazy/nuts/insane construction, which we can only process and understand if we look up some further information at the level of the SUBJ VERB OBJ ADJP construction. All of this triggers a surge of upward activation, and it does not stop there either. In order to process the SUBJ VERB OBJ ADJP construction, the hearer has to look up information that is represented at an even higher level of abstraction, namely, at the level of the Subject Predicate construction, which tells us that subjects can occur with verb phrases and that this yields a complete sentence of English. The complete inheritance view makes a very strong prediction here and I would argue that it is a questionable prediction. Do we really have to access the Subject Predicate construction, a very abstract schema, if we hear someone say, You drive me crazy?

So what is wrong with the naive strengthening view? I think there are some examples that illustrate why we should be skeptical. There are some linguistic units for which it is doubtful that they strengthen any constructions that are more abstract. An example for that would be linguistic units that simply do not have overarching categories, so that there is no abstract schema to send activation to. Let me give you an example from my native language, German. When a child sneezes, the parent may comment that by pronouncing the word hatschi. That is an onomatopoeic word that echoes the sound of a sneeze. In English, you might respond to a sneeze by saying blessyou. The social routine is similar, but the words are not the same. I would say that hatschi is a word in German, but do not ask me what kind of word it is. Is it a noun? There are perhaps arguments for that analysis, but in any event hatschi is not a very prototypical noun. When someone says hatschi, I have grave doubts that hearers will activate of general noun construction in German.

Let's take a different example of that sort, hallelujah. What kind of word is hallelujah? You might say that it is an exclamation. There are others like damn $i t$, which consists of a verb and a pronoun, but which is not really a sentence either. These are problem cases. These are linguistic units that are impossible to categorize. Whenever a word is very difficult to categorize, I find it highly unlikely that we look up some kind of information that is stored in a more abstract category to understand that very item.

De-categorialized units represent another type of example. When lexical items grammaticalize, they shed some of their original category features. The word long is an adjective in "as long as a python", and a part of a clause connector in as long as you do not get caught. When I say as long as you do 
not get caught, do hearers activate and strengthen the English adjective? In as long as, the adjective long has been de-categorialized. It no longer instantiates its upward link with the category adjective, so it can't send any activation that way.

The last type of example I'd like to mention concerns highly frequent constructs, as for instance the phrase Oh my God. That phrase contains the noun God, but it is debatable whether Oh my God sends activation to the English noun construction. I do not think so, because Oh my God has become entrenched as a holistic unit. When a string of words becomes entrenched as a holistic unit, the parts become increasingly hard to access by themselves.

All of this, in my view, casts doubt on the complete inheritance view. I think it is much more plausible to assume that information is stored locally, redundantly in the case when there are still connections to the higher levels, but non-redundantly if there are no connections, if those connections are cut as in the case of de-categorialized or highly frequent units.

Let me ask the question the other way around. Which structures do trigger upward strengthening? There are three points that I would like to make here. Expressions that you hear trigger upward strengthening if three conditions are met. Linguistic units require you to activate higher levels in the constructional network if they contain a strong cue for an overarching generalization, if the construct is infrequent and if the construct is not very similar to already known instances of the overarching generalization.

I would like to illustrate this point with another non-linguistic example. Let's say that you observe an unusual cat. Cats can be seen every day and they all look fairly similar, with fur, a tail, and four paws. Suppose that you see one that has no fur, ears that look a bit bigger than usual, and eyes that are bright blue. This kind of stimulus would force you to reconsider a category that you have, an abstract schema of cats in this case. That is the theoretical foundation of what I will have to say about the upward strengthening hypothesis.

Since it is a hypothesis, I want to test it. I want to test the idea that stimuli of this kind actually have the effect of strengthening an existing category. I will do that on the basis of diachronic corpus data. There is a particular case study that I want to discuss, namely, English noun-participle compounds. I have mentioned that construction type a few times already. It is instantiated by forms such as doctor-recommended or chocolate-covered.

There are three questions that I would like to ask with regard to these nounparticiple compounds. First of all, how have they changed chronically? It has been pointed out that this construction, noun-participle compounding, is related to the passive construction in English. I wanted to know whether the two constructions have changed in comparable ways. Are the developments 
in the compounding construction related to changes that have been going on in the passive? Finally, I want to use the empirical findings that emerge here for theoretical considerations that concern the upward strengthening hypothesis. Specifically, I will examine whether upward strengthening can be profitably viewed as a process grammaticalization, and if not, what else this phenomenon might be.

Let me give you a quick overview of the rest of this lecture. First, I will discuss how we can imagine the relation between noun-participle compounding and the English passive. Based on that discussion, I will present findings from a diachronic corpus analysis that explores how the compounding construction has changed and whether these changes are paralleled by changes in the passive. Then I will raise the question of whether what we see here can be brought under the umbrella of grammaticalization.

What are English noun-participle compounds? Huddleston and Pullum (2002) in The Cambridge Grammar of English describe this construction and they offer examples such as drug-related, home-made, safety-tested, and taxpayer-funded. The construction is a very productive, very common pattern of English grammar. Huddleston and Pullum state that these compounds generally correspond to syntactic passives with a prepositional phrase. Drug-related can be paraphrased as related to drugs, home-made means made at home, safety-tested means tested for safety and taxpayer-funded means funded by taxpayers.

The noun in this construction can instantiate the agent of an action that would be represented by the by-phrase of a passive construction. However, there are other roles. In taxpayer-funded, the noun instantiates an agent, so something was funded by the taxpayer. The noun can also instantiate a location. The word home-made does not refer to something that was made by the home, it refers to something that was made in the home or at home. In drugrelated, the noun expresses a cause, so something is caused by drugs, and in safety-tested, the noun expresses a purpose, so something was tested for the purpose of safety. This suggests that there are very few constraints on the roles that can be expressed in the noun of a noun-participle compound.

However, there is one rather fundamental constraint, which is identified by Bauer and colleagues (2013) in the Oxford Reference Guide to English Morphology. Bauer and colleagues state that "The first element cannot receive an object interpretation." In the compound doctor-recommended, the doctor would be the subject of an action. In arsenic-exposed, someone was exposed to arsenic. The noun would correspond to a prepositional object of the verb, not its subject. Then, there are examples that do not work, as for instance luncheaten. You cannot say “* The lunch-eaten, participants returned to the conference", 
even though that would be a meaningful concept. I mentioned this in the first lecture that constraints of this kind allow you to identify that something is a construction. Here we have an example of this. There is a constraint that we can call the no-object constraint, which would be one piece of evidence suggesting that what we are looking at here is really a construction, something that you have to learn. I hope you agree that the no-object constraint is something of a puzzle. Why is it that the direct object is the only grammatical relation that does not work in this construction? Some work has been done on this particular problem.

One explanation has been offered by Rochelle Lieber (1983), who argues that the no-object constraint in noun-participle compounding is the same phenomenon that we see in the passive, namely that an object is promoted to the role of the subject. Consider an active sentence such as "The dog bit the man". In the passive, the man appears in the subject position, "The man was bitten by the dog". Lieber (1983) argues that this is something that we also see in nounparticiple compounding. She argues that the past participle "expels" or drives out the direct object from its ordinary position. This happens in both the passive and a noun-participle compounding. In the passive, the object of a transitive verb is no longer part of the verb phrase in which it originally appears. When I say "The strawberries were picked by hand", the strawberries appear outside the verb phrase. The verb pick has a direct object, you pick the strawberries, so the strawberries are part of the verb phrase in a canonical active sentence. In the passive, the object no longer appears in its original phrasal environment. Instead, it appears as the grammatical subject.

In noun-participle compounding, we see something very similar. The object of a transitive verb is no longer part of the compound phrase itself. I can say something like the hand-picked strawberries, where the strawberries are no longer part of the construction that contains the verb. This is parallel to the passive. In both cases, we have an object that is evicted, that is driven out of its place of origin.

Lieber (1983) presents a syntactic tree that is meant to illustrate this. She posits argument structure traits that are thought to move upwards in the syntactic tree and that cause the no-object constraint. The governing node imposes selection restrictions, which determine what can occur in this kind of phrase. What it states is that there should be no direct object in its scope. Cognitive linguists may view this syntactic representation with some skepticism, but actually the idea of inheritance makes use of the same idea. Inheritance means that abstract schematic construction impose restrictions on concrete constructs. Generative linguistics and construction grammar are really not that far apart from each other in that particular respect. Both the passive construction 
Lieber (1983)

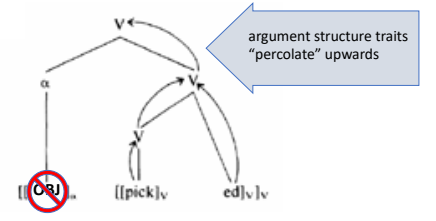

FIGURE 1

and noun-participle compounding inherit the argument structure of the past participle, which prohibits any direct object in its domain. But what are the implications of this?

What does it mean that both noun-participle compounding and the passive inherit the no-object constraint from the participle? It could mean that there is a higher-order schema, i.e. an overarching generalization. It could also mean that speakers treat them as two separate constructions, so that both constructions inherit characteristics from the participle, but are still represented individually.

In earlier lectures, I have talked about the controversy of higher-order schemas. Before we return to these two hypotheses, let us look at empirical data.

For this analysis, I used the сон one more time. I searched the сон for noun-participle compounds. I implemented several search criteria that gave me the examples that I was looking for.

The forms that I retrieved were able-bodied, age-old, absent-minded, air-conditioned and so on and so forth. Not all of them are target cases. For example, old is not a participle, so I weeded out those cases manually. I ended up with a dataset that consists of about 31,000 types, that is, different nounparticiple compounds such as god-abandoned, tax-abated, self-absorbed, man-abused and so on. Across those 31,0oo types, the dataset contains about 150,00o tokens. Many types are only represented in one or two decades. What can we learn from this dataset?

A first observation that I would like to share with you is that the construction quadruples in text frequency over the past two centuries. Noun-participle compounding is a success story in the recent history of English grammar.

This increase in token frequency is accompanied by an increase in the variety of types that are used. There are more and more different noun-participle 


\section{increase in token frequency increase in type frequency}

FIGURE 2

compounds that people are using. In other words, the construction is developing. In the rest of the time, I will be concerned with the question of what exactly this development is.

How does the construction change with regard to the participles that are found in it? I was interested in the following questions. How does the increase in type and token frequencies come about? Which are the participles that carry the increase?

The types that I retrieved from the сонA form families of different sizes around the same participle. A small family of this kind is arranged around the participle wetted, "made wet by something". The database contains compounds such as dew-wetted, gall-wetted, snow-wetted and tear-wetted, "made wet by tears". You understand the concept, but I very much doubt that you've heard this word very often. The participle yellowed occurs in the compounds age-yellowed,fear-yellowed, opium-yellowed, or smoke-yellowed. This is a slightly larger family. A large family is illustrated by the participle coated. Compounds with that participle include aluminum-coated, bearskin-coated, beech-coated, blood-coated, candy-coated, caramel-coated, carbon-coated, cement-coated, and many others.

I would like to show you how the noun-participle compounding construction changed with regard to its participle families. This graph shows the participle families that were most frequent in the early 19th century. Each bubble represents a participle family, and the bubbles are positioned with respect to their normalized type frequency on the $y$-axis, and with regard to their combined token frequency on the $x$-axis. If a participle appears high up, that means that it occurs with many different nouns. If a participle appears far to the right, it means that there are many tokens that involve that participle, regardless of how many types there are. Bubble size also corresponds to token frequency, so 

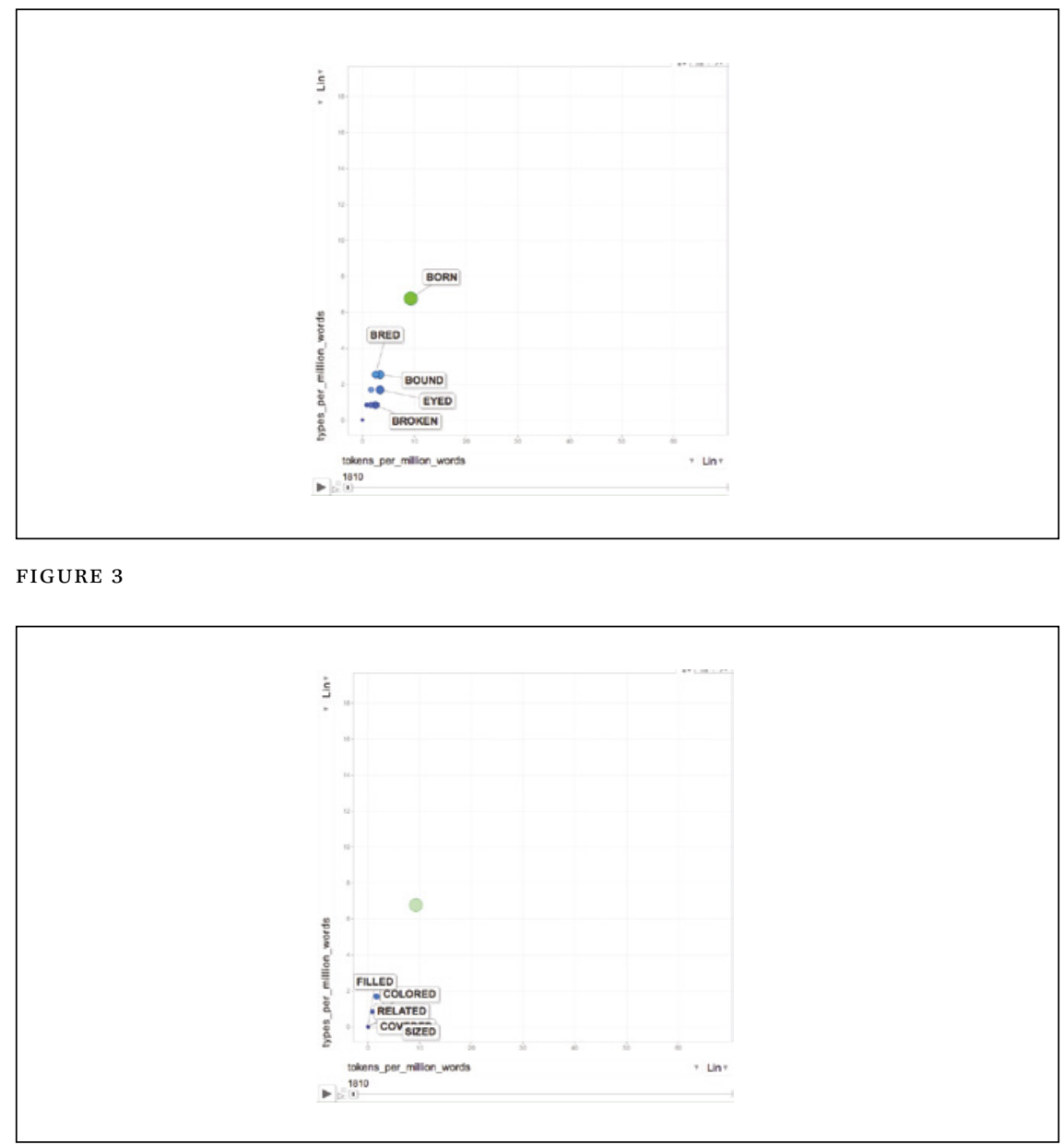

FIGURE 4

large bubbles account for many instances in the corpus. Among the participles with the highest type and token frequencies, we find born as in Alaska-born, bred as in home-bred, bound as in Britain-bound and then eyed and broken, as in eagle-eyed or heart-broken. We'll talk about those forms in a minute. For now, what I would like you to do is watch how these participle families have developed over the years. We start in the year 1810 and finish in the first decade of the 2ooos.

In the first decades, there are small and unsystematic changes, up to the beginning of the 2oth century. Then there are several participles that become more frequent. In the second half of the 2oth century, there is one participle 


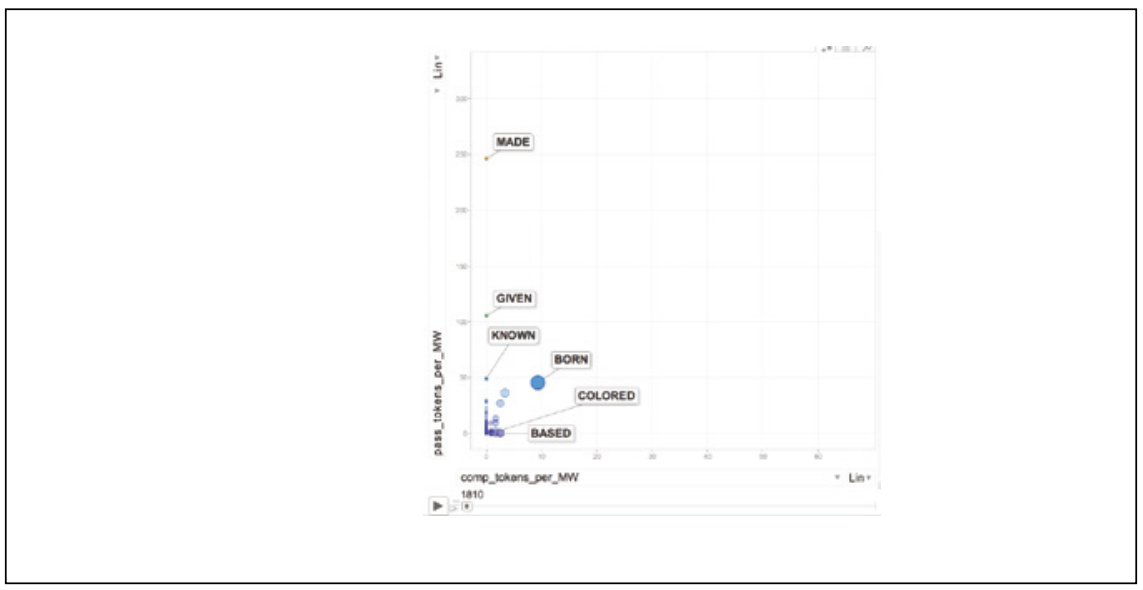

FIGURE 5

family that breaks out of the field and makes its way up to the upper right, where it has many different types and is represented by very many tokens.

Let's look at the same development again. This time, I have marked up the participles that turn out to be very successful in the late 2oth century. Again, we see some back and forth in the first decades. Then colored and shaped emerge and stay in the vanguard of this field for quite a while. Then in the 195 os and 196os, we have a few late-comers that join the field. By the 1970s, it is the participle based that escapes from the rest of the field and emerges as the most frequent participle in terms of types and tokens. You see that it draws the entire field with it to a certain extent. If we compare the 1810 s to the 200 os, we see a clear difference.

Let me summarize the development. The participles change. In the 181os, frequent participle include born, bred, bound, and eyed as in Harvard-bred or context-bound. In the 19oos, colored, shaped and covered dominate. In the 2ooos, frequent participles are based, related and sized, as in the compounds Houston-based, work-related, and toddler-sized.

As I said earlier, one of my leading questions was whether or not nounparticiple compounding would show diachronic developments that are similar to those of the passive. If for instance, passive uses of the verb relate increased diachronically, then the increase of forms such as work-related would be just an epiphenomenon of the history of the word relate, not a consequence of anything happening to the compounding construction.

How does the compounding construction compare to the passive? I went back to the сона and retrieved examples of the passive, specifically passages where a form of to be is followed by a past participle. This covers only a subset of all passives, but a substantial subset. I retrieved three million examples that 
fall into about 5000 different participle types. I identified participle types that occurred both in noun-participle compounding and in the passive, and visualized that data in order to see whether the overlapping types show similar frequency developments.

This chart shows the participle types arranged according to their token frequency in the passive, which is shown on the $y$-axis, and token frequency in the compounding construction, shown on the $x$-axis. Color represents passive token frequency, and size represents compound token frequency. Before we watch what happens here, let me discuss what I expected to see. If the passive and the compounding construction behave in similar ways, then the participles should move one step on the $x$-axis and one corresponding step on the $y$-axis. We should see diagonal developments in some way or other, either both increase or both decrease. If on the other hand, both constructions develop in relative autonomy, we should see movements that are either just horizontal or vertical.

The result is the latter. There are clearly no diagonal developments. Most of the changes that you see are either on the horizontal plane or on the vertical plane. The horizontal movement of based is clear to see. We further see a vertical fall of made, which indicates a frequency decrease in the passive.

The data indicate that we are observing independent developments. Changes in noun-participle compounds are independent of changes that happen in the be-passive, and the participle types that stand out most in the history of noun-participle compounding do not correspond to passive sentences. Sentences like "The company is based in Houston", do not have an active counterpart. "Houston bases the company" is not a grammatical sentence of English. The same goes for "The problem is related to drug abuse" or "The car is sized just right". This speaks for hypothesis B that I presented earlier. It suggests that there is no higher order schema for noun-participle compounding and the passive, but instead two independent generalizations.

With that, I would like to come to the theoretical part of my argument. Is upward strengthening grammaticalization? In earlier lectures, I used Hopper and Traugott's (2003) definition of grammaticalization: "The change whereby lexical terms and constructions come in certain linguistic contexts to serve grammatical functions, and, once grammaticalized, continue to develop new grammatical functions."

Having adopted this definition, let me elaborate on grammatical functions and what they are. I have discussed procedural meaning before, which expresses who did what to whom, when something happens and how the elements of clause are related. I view noun-particle compounding as an argument-structure construction, very much like the ditransitive construction that Adele Goldberg has worked on. Goldberg (1995: 39) has formulated 


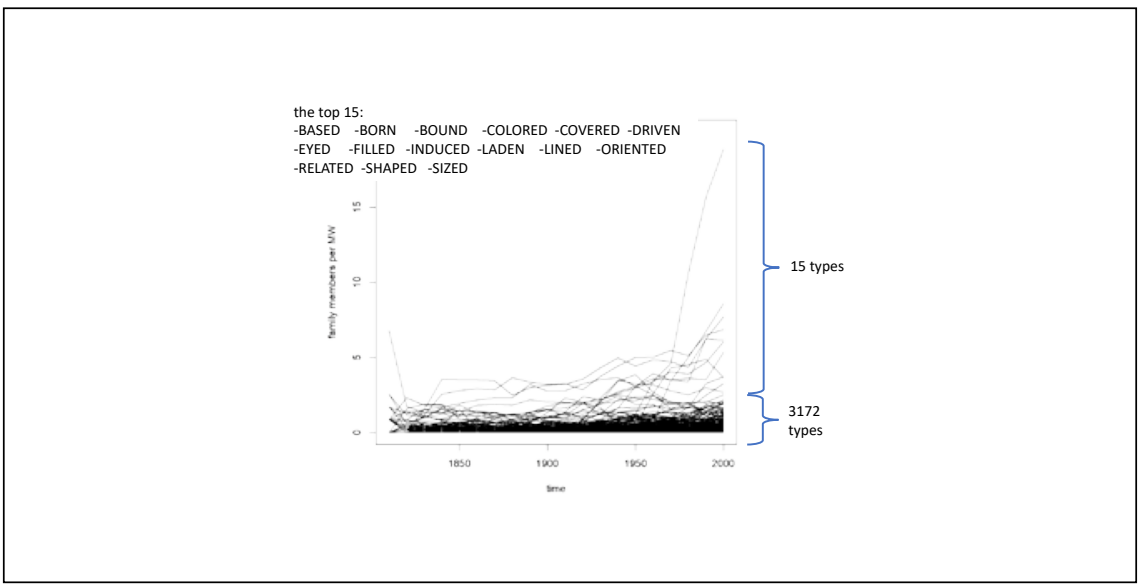

FIGURE 6

the "scene-encoding hypothesis", the idea that basic morphosyntactic patterns correspond to basic recurrent patterns of human experience. I think the meaning of noun-participle compounding fits reasonably well into that idea.

I have shown that noun-participle compounding has undergone dramatic frequency increases. We have a pattern with a grammatical function that is apparently on the move. Does that mean that we are looking at a process of grammaticalization? I am actually not convinced that this is the case, and I would argue against it in the following way.

This graph visualizes the development of the participle families in nounparticiple compounding in a static way. It is based on the same data that you have seen before. Each line is a participle family, and you can see for each decade how prolific that family is. The sharply increasing line at the end represents the participle-based. What is interesting is that the terrific frequency increase of the construction is carried by a very small set of participle families, 15 families to be exact, -based, -born, -bound, -colored, -covered and a few others. The other 3000 types are the many infrequent participle families that did not contribute substantially to the frequency increase. This leads me to formulate the following points.

The increase in type and token frequencies is not carried by a broad base of different lexical items, only by a small "elite". The developments do not show systematic relaxation of constraints, so the no-object constraint is still there. Agents, instruments, causes, etc. are found in the noun slot at all times. When we consider morphosyntactic form, there is no formal change in the pattern as such. There is host-class expansion to some extent, but not much either. If what we see is not grammaticalization, it is reasonable to ask what else it might be. 


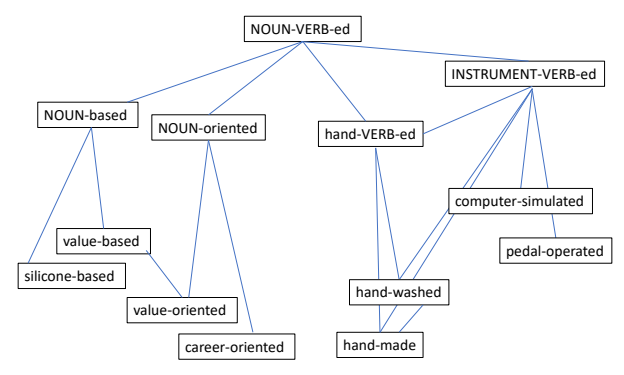

FIGURE 7

I view this as an instance of constructional change. Noun-participle compounding is a construction, a generalization that speakers make about formmeaning pairs in language use. Over time, this construction changes. Certain patterns become relatively more prominent and "emancipate" themselves. We can say that a pattern like NOUN-oriented emerges, and we have words such as relationship-oriented or career-oriented that illustrate that. Others sub-patterns decrease in relative prominence and become less productive. There is a participle in English with the form stricken. It is found in compounds such as panicstricken, affected by panic, and woe-stricken, affected by emotional pain. That participle is no longer productive, so that node in the network has faded away. I like to think of this as change in a network of constructional patterns, where a lot of strengthening affects lower levels of the network, but crucially, there is no strengthening of the top node, so that the general noun-participle construction does not receive any upward strengthening.

This slide shows the kind of network that we can imagine. At the top of the network, we have the very abstract generalization of noun + participle. Then there are more concrete instances of this, like Noun-based or Noun-oriented, or hand-participle like hand-washed, hand-made, or Instrument-participle like computer-simulated or pedal-operated. There are generalizations at all levels of abstractions, and some are strengthened, some are weakened. Overall, this highest node does not become any stronger over the course of the time period that I have investigated.

One last point that I want to address is how we can model that kind of change in a constructional network. Diachronic corpora show us which instances of a construction are used during a given period of time. Changes in the instances that come in lead to strengthening of subconstructions and potentially the overarching construction. Let me come back to the example of the strange-looking cat. Each usage event potentially can cause some upward 
strengthening, and the amount of upward strengthening depends on cue validity, frequency, and salience.

Cue validity relates to the following question: Is the usage event easily categorized as a combination of noun and past participle? If categorization is easy, then there would be more upward strengthening. Something like chocolatecovered where -covered is clearly recognizable as a participle, this sends a lot of activation. We can contrast that with the participle -stricken, which is not easily recognized as a participle. As a consequence, there would be less activation and less upward strengthening. The form -eyed is not even a true participle, so there would be even less activation.

Frequency captures how often hearers encounter linguistic usage events. I have discussed patterns like "Oh my god" or "Damn it", or "Thankyou". Frequent forms emancipate themselves from overarching schemas, which leads to less upward strengthening. Conversely we can argue that the lower the frequency, the more upward strengthening should occur. Take an example such as garlicinfused. The concept is clear, but the word itself is infrequent. That sends a lot of upward strengthening. Aluminum-coated is more frequent, so it would trigger less upward strengthening. The compound oil-based is even more frequent and would be hypothesized to trigger an even smaller amount of upward strengthening.

Salience relates to the similarity between a usage event and other usage events of the same category. A compound such as oven-roasted would be similar to the form oven-baked. It is not very unusual in that regard. The reasoning would be that more unusual items send more activation to the category. The compound war-acquired is not very similar to previously seen compounds, so it sends more activation to the overarching construction. The compound baconwrapped is not so unusual and would therefore not trigger as much upward activation.

I computed a measure of upward strengthening over time on the basis of the соHA data. In each decade, constructs appear in language use. These constructs create the maximal amount of upward strengthening if they are new, if they contain a recognizable participle, and if they are relatively low in frequency, and if they are semantically different from the rest.

How did I define semantically different? This relates to concepts from distributional semantics that I discussed yesterday.

I created a semantic vector space for the participles in the database, which left me with a semantic space like the ones that you saw yesterday. The idea would be that participles from a sparsely populated area would result in more upward strengthening than participles that we find in the center, which is more densely populated. In this graph, sparse areas are shown in yellow. The darker areas of orange and red are more densely populated. 
- participles from a "sparse" area result in more upward strengthening than participles from a "dense" area

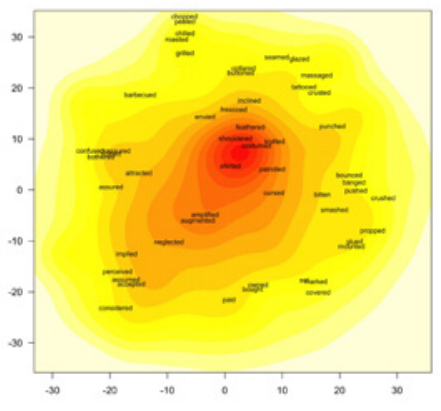

FIGURE 8

Constructions can also be forgotten. Generalizations that are not strengthened undergo decay if no upward strengthening occurs. My modeling thus included a decay function. When a construction is not regularly updated, then it is eventually forgotten.

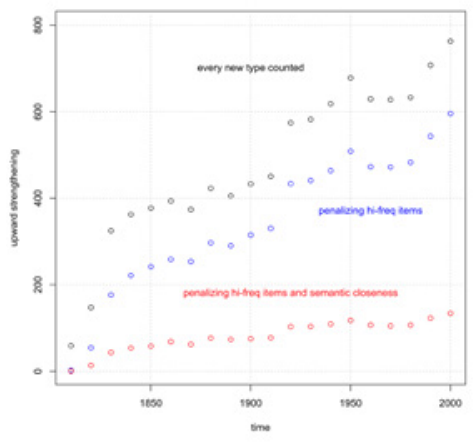

FIGURE 9

On the basis of these measurements, I created the graph you see on this slide. The graph is based on the following information. First, I determined for each period of time how many new instances are attested. I also determined how saliently they illustrate the category and how similar they are to already existing types. Based on these factors, I calculated a measure of upward strengthening. If every new type is counted, without adjusting for frequency or salience, then the result is a strongly increasing curve that would suggest a lot of upward strengthening. If high-frequency forms are penalized, so that they send less 
activation to the higher node, the curve is already a little bit flatter. When highfrequency items and semantic closeness are penalized, the resulting curve is just about flat. That would imply that the highest node in the network does not receive any amount of upward strengthening.

This would explain why I do not see this as a case of grammaticalization despite increases in type and token frequencies. If we do not attribute upward strengthening to types that are not clear members of the participle category, if we do not attribute upward strengthening to types that are highly frequent like road-tested, and if we do not attribute up strengthening to types that are semantically similar to already existing types, then we can motivate why speakers do not strengthen their overarching generalization over all noun-participle compounds.

Asked the other way around, what new types would lead to upward strengthening, and thus to grammaticalization?

According to what we know about grammaticalization and about known cases of grammaticalization, upward strengthening would occur when speakers continually produce new types which clearly instantiate the construction, and which are semantically dissimilar to already existing new types. That is, if speakers always try to stretch the limits of what a construction can do, and if they produce instances that are a little different from what is already known, that would result in upward strengthening and in grammaticalization. Once a construction is grammaticalized to a high degree, then upward strengthening should naturally cease to increase.

I am summing up here. I have discussed grammaticalization as a specific type of change in the network of constructions. I have invited you to think about that kind of change in terms of gradient strengthening of nodes and connections in that network. How do nodes in the upper layers of the network come into being and how are they subsequently strengthened? That is, I think, an important idea to think about. The upward strengthening hypothesis is a part of a more general enterprise that tries to understand grammaticalization in constructional terms. Like other issues that I have been discussing, as for example higher-order schemas, I think that this is an area that requires further research. With that, I would like to come to an end. Thank you for your attention once more. 